(2) Open Access Full Text Article

\title{
Downregulation of PDIA3 inhibits proliferation and invasion of human acute myeloid leukemia cells
}

This article was published in the following Dove Press journal:

OncoTargets and Therapy

\author{
Qidong Ye' \\ $\mathrm{Pan} \mathrm{Fu}^{2}$ \\ Jiaying Dou ${ }^{2}$ \\ Nina Wang ${ }^{2}$ \\ 'Department of Pediatrics, Ningbo \\ First Hospital, Ningbo Hospital of \\ Zhejiang University, Ningbo, People's \\ Republic of China; ${ }^{2}$ Department of \\ Hematology, Shanghai Children's \\ Hospital, Shanghai Jiao Tong University, \\ Shanghai, People's Republic of China
}

Correspondence: Qidong Ye Department of Pediatrics, Ningbo First Hospital, Ningbo Hospital of Zhejiang University, No 59 Liuting Street, Ningbo 315000 , People's Republic of China Tel +8657487085588 Email qidongye7806@sina.com
Introduction: Acute myeloid leukemia (AML) is a common malignancy of the hematopoietic system. In bone marrow samples of AML patients, PDIA3 expression was higher than that in the samples of healthy controls. We aimed at exploring the effect of PDIA3 siRNA on proliferation, apoptosis, migration, and invasion of AML HL-60 and HEL cells.

Materials and methods: RT-PCR was performed to identify PDIA3 expression. Cell proliferation was assessed by MTT. Flow cytometry analysis and transwell were used to detect cell apoptosis, migration and invasion. Gene set enrich-ment analysis (GSEA) was employed to explore the PDIA 3-associated pathways in AML. Western blotting was used for protein expression detection.

Results: PDIA3 siRNA significantly inhibited the proliferation of AML cells at 24 and $48 \mathrm{~h}$. PDIA3 siRNA notably enhanced the percentage of apoptotic cells. The migration and invasion abilities of HL-60 and HEL cells in the PDIA3 siRNA group were significantly suppressed compared with those in the control and siNC groups. GSEA of the Cancer Genome Atlas dataset showed that Kyoto Encyclopedia of Genes and Genomes oxidative phosphorylation and amino sugar and nucleotide sugar metabolism pathways could be correlated with PDIA3 expression; this was further confirmed in AML cells by Western blotting. MAPK signaling was also blocked by PDIA3 siRNA.

Conclusion: PDIA3 siRNA effectively enhanced apoptosis, and suppressed proliferation, invasion, and migration of AML cells by regulating oxidative phosphorylation and amino sugar and nucleotide sugar metabolism pathways, and MAPK signaling, which can provide novel therapeutic targets for AML.

Keywords: PDIA3, acute myeloid leukemia, migration, invasion, gene set enrichment analysis, MAPK pathway

\section{Introduction}

Acute myeloid leukemia (AML) comprises a heterogeneous group of hematological malignancies characterized by expansion of clonal myeloid blasts in the bone marrow, blood, and other tissues. About 21,380 new AML cases and 10,590 deaths from this disease were reported in 2017 by the American Cancer Society. ${ }^{1}$ Despite the advancements in modern chemotherapy, the prognosis of patients with AML has remained poor. $^{2,3}$ Identifying the mechanisms involved in AML metastasis may lead to innovative treatment methods and improved patient outcomes.

PDIA3, known as ERp57 or glucose-regulated protein, has a molecular weight of $58 \mathrm{kDa}$ and is a thiol-oxidoreductase chaperone belonging to the protein disulfide isomerase (PDI) family. ${ }^{4}$ PDIA3 expression has been observed in various types of human cancers, including ovarian, mammary, uterine, pulmonary, and gastric cancers, and hepatocellular carcinoma. ${ }^{4}$ It is reported that PDIA3 expression is significantly increased in hepatocellular carcinoma, and high PDIA3 expression is associated with 
cancer cell proliferation and poor prognosis. ${ }^{5}$ PDIA3 is also highly expressed in a newly established serous ovarian cancer cell line, YDOV-139. ${ }^{6}$ PDIA3 expression is increased in primary ductal breast cancer with lymph node metastasis. ${ }^{7}$ However, no studies have yet demonstrated the exact role of PDIA3 in AML, and the pathways that signal PDIA3 to exert its function have not been elucidated. Consequently, a possible involvement of PDIA3 subfamily in the development and progression of AML, a malignancy originating from the hematopoietic system, was proposed.

We previously found that PDIA3 expression was remarkably increased in AML patients. Therefore, in this study, we focused on the effect of PDIA3 siRNA on the migration and invasion in HL-60 and HEL cells, which markedly expressed PDIA3. Moreover, the underlying mechanisms have been investigated to provide a basis for the treatment of AML.

\section{Materials and methods Patients and tissue samples}

Bone marrow cells were collected from 20 patients newly diagnosed with AML admitted to Ningbo First Hospital of Zhejiang University. Twenty healthy bone marrow donors served as normal controls. Preoperative clinical and pathological follow-up data were completed by all the patients. Written informed consent was obtained from the study subjects before the use of these clinical samples, and the study protocol was approved by the ethics committee of Ningbo First Hospital of Zhejiang University.

\section{Cell culture}

6TCM, HL-60, K-562, THP-1, HEL, and A3 cells were obtained from the Shanghai Cell Bank, Chinese Academy of Sciences (Shanghai, People's Republic of China) and cultured in RPMI medium (St Louis, MO, USA) supplemented with $10 \% \mathrm{FBS}$ and $1 \%$ penicillin/streptomycin at $37^{\circ} \mathrm{C}$ in a humidified atmosphere of $5 \% \mathrm{CO}_{2}$.

\section{siRNA transfection}

For siRNA transfection, HL-60 and HEL cells, which were selected for transfection due to high PDIA3 expression confirmed by Western blot, were seeded into 12-well tissue culture plates at a density of $6 \times 10^{4}$ cells/well. The PDIA3 siRNA or control siRNA (GenePharma, Shanghai, People's Republic of China) were then transfected into cells by using Lipofectamine 2000 (Thermo Fisher Scientific, Waltham, MA, USA) according to the manufacturer's protocol. After $48 \mathrm{~h}$, the transfected cells were collected and processed for subsequent experiments.

\section{Cell proliferation}

Cell proliferation was evaluated by 3-(4,5-dimethyl-2thiazolyl)-2,5-diphenyl-2 $H$-tetrazolium bromide (MTT) assay. Cells were seeded into 96-well plates at a density of 2,000 cells per well and cultured for indicated times, followed by incubation at $5 \% \mathrm{CO}_{2}$ and $37^{\circ} \mathrm{C}$ following the manufacturer's instructions. Absorbance was measured at $490 \mathrm{~nm}$ using a microplate reader absorbance test plate (Molecular Devices, San Jose, CA, USA). Cells were detected from three wells per group.

\section{Annexin V-propidium iodide apoptosis detection}

The cell apoptosis rate was detected by flow cytometry using an Annexin V-FITC apoptosis detection kit (Nanjing Keygen Biotechnology Co. Ltd., Nanjing, People's Republic of China). Following 48-h transfection, the cells were collected, washed with ice-cold PBS, and then resuspended in $500 \mu \mathrm{L}$ binding buffer at a concentration of $1 \times 10^{6}$ cells $/ \mathrm{mL}$. Subsequently, $5 \mu \mathrm{L}$ Annexin V-FITC was added and incubated for $10 \mathrm{~min}$ at room temperature. After addition of $5 \mu \mathrm{L}$ propidium iodide, the cells were analyzed using a FACSCalibur flow cytometer (Becton Dickinson, Franklin Lakes, NJ, USA).

\section{Cell invasion and migration assay}

Invasion and migration activity of HL-60 and HEL cells were determined using a 24-well transwell chamber coated with or without Matrigel (Becton Dickinson) on the upper surface of the membrane with $8 \mu \mathrm{m}$ pore size (Sigma-Aldrich). Briefly, the transfected cells $\left(1 \times 10^{4}\right.$ cells/well $)$ were added in a volume of $100 \mu \mathrm{L}$ serum-free medium to the upper transwell chamber. The lower chamber was filled with medium containing 10\% FBS. After 24-h incubation, the cells that had invaded or migrated to the lower surface of the filter were counted visually under a microscope (OLYMPUS).

\section{Western blot analysis}

Protein concentrations were determined using a BCA protein assay kit (Thermo Fisher Scientific). An equal amount of proteins per sample was loaded and separated by $10 \%$ sodium dodecylsulfate-polyacrylamide gel electrophoresis (SDSPAGE), followed by electrotransfer onto a polyvinylidene fluoride (PVDF) membrane (Millipore, Bedford, MA, USA). Following blocking with 5\% skimmed-milk powder in Trisbuffered saline containing $0.05 \%$ Tween- 20 for $1 \mathrm{~h}$, the membranes were incubated with antibodies specific for MAT1, caspase-9, RhoC, CDK1, phosphorylation (p-) P38, P38, p-JNK, JNK, p-extracellular regulated protein kinase (ERK), ERK, PCNA, and GADPH overnight at $4^{\circ} \mathrm{C}$. Subsequently, 
the membranes were washed and incubated with 1:1,000 dilutions of horseradish peroxidase-conjugated secondary antibodies for $1 \mathrm{~h}$. The bands were visualized using enhanced chemiluminescence detection kit (Santa Cruz Biotechnology, Dallas, TX, USA).

\section{Bioinformatics analysis}

The gene set enrichment analysis (GSEA) of pathways and genes was performed based on the Cancer Genome Atlas (TCGA) dataset using the GSEA version 2.0 from the Broad Institute at MIT. In this analysis, the gene sets of fewer than 10 genes were excluded. The $t$-statistic mean of the genes was computed in each Kyoto Encyclopedia of Genes and Genomes (KEGG) pathway. Using a permutation test 1,000 times, $P \leq 0.01$ was chosen as the significance level cutoff for the most significant pathways related to PDIA3 expression.

\section{Statistical analysis}

All results are presented as the mean \pm SD of three independent experiments. Data for multiple comparisons were subjected to one-way analysis of variance with SPSS version 13.0 followed by Dunnett's test. $P<0.05$ was considered statistically significant.

\section{Results}

\section{High PDIA3 expression in the bone marrow of AML patients as well as in AML cell lines}

We first identified PDIA3 expression in the bone marrow of AML patients and controls by RT-PCR. As displayed in Figure 1A, compared with that of normal control, the bone marrow of AML patients showed a remarkable increase in mRNA expression of PDIA3 $(P<0.05)$, suggesting that overexpression of PDIA3 may be involved in the initiation and/or progression of AML.

PDIA3 expression was also examined in AML cell lines by RT-PCR and Western blot analysis. As shown in Figure $1 \mathrm{~B}$ and $\mathrm{C}$, results from RT-PCR and Western blot showed that mRNA and protein levels of PDIA3 in HL-60 and HEL cells were higher than those in other cell lines.
A

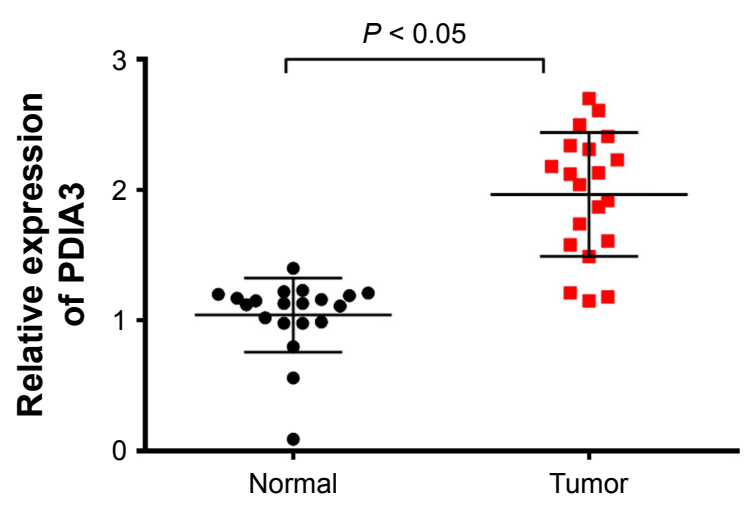

C

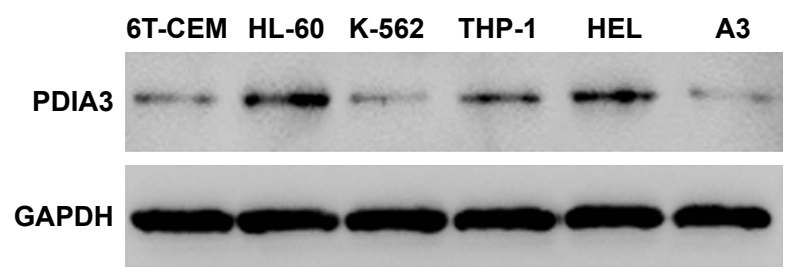

B
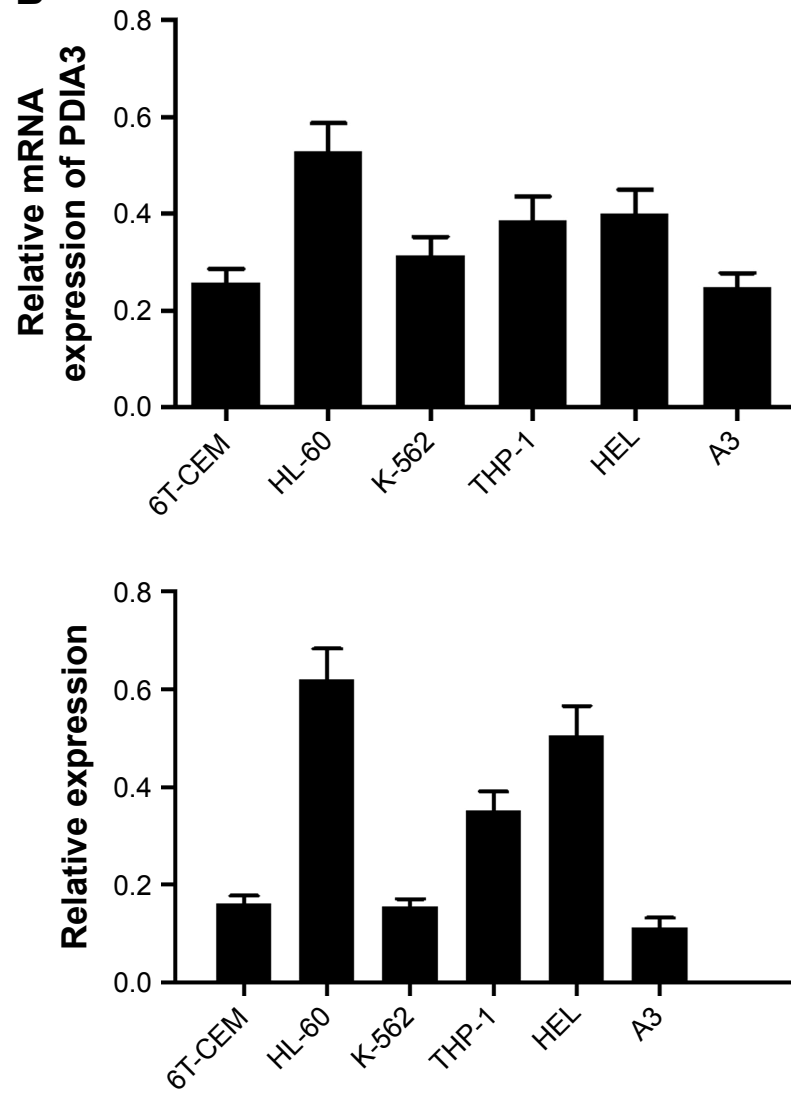

Figure I PDIA3 expression in 20 bone marrow tissues of acute myeloid leukemia (AML) and normal tissues.

Notes: (A) Twenty bone marrow tissues of AML patients were collected and 20 healthy bone marrow donors served as normal controls. The mRNA expression of PDIA3 was identified by RT-PCR. PDIA3 expression was measured by RT-PCR (B) and Western blot (C) in 6T-CEM, HL-60, K-562, THP-I, HEL, and A3 cells. Data were presented as mean $\pm S D, n=6$.

Abbreviation: GADPH, glyceraldehyde 3-phosphate dehydrogenase. 
As a result, HL-60 and HEL cell lines were used to conduct further investigations.

\section{Effect of PDIA3 siRNA on apoptosis of HL-60 and HEL cells}

PDIA3 mRNA was interfered in HL-60 and HEL cell lines as previously described. The interference efficiency was then identified by RT-PCR and Western blot analysis. RT-PCR and Western blot analysis showed that protein levels declined dramatically in PDIA3 siRNA group in both the cell lines compared with the control and mock groups (Figure 2A and B). Cell proliferation was then determined by MTT assay. As shown in Figure 2C, the viability of both
HL-60 and HEL cells in the siRNA group was significantly decreased at 24 and $48 \mathrm{~h}$ compared with the control and siNC groups $(P<0.01)$.

\section{PDIA3 siRNA induced apoptosis and inhibited invasion and migration of HL-60 and HEL cells}

After $48 \mathrm{~h}$ of transfection, cell apoptosis was analyzed using flow cytometer. As depicted in Figure 3A and B, compared with the control and siNC groups, the PDIA3 siRNA group displayed a significant increase in the percentage of apoptotic HL-60 and HEL cells in all the phases $(P<0.01)$.
A

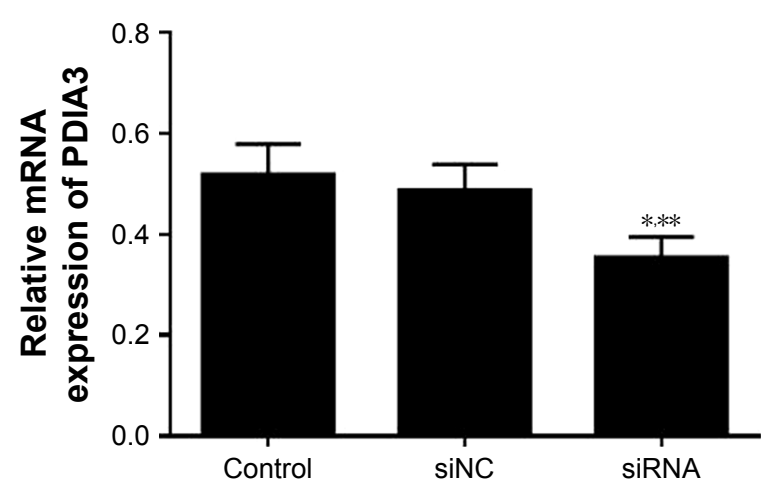

B

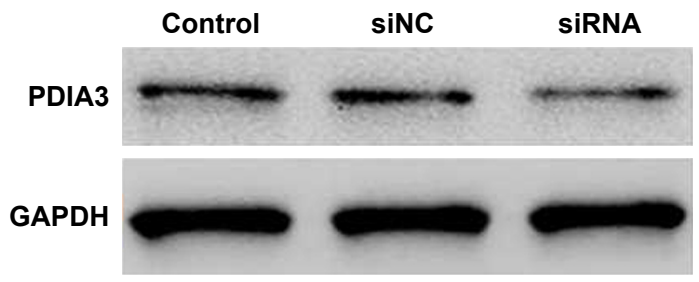

C

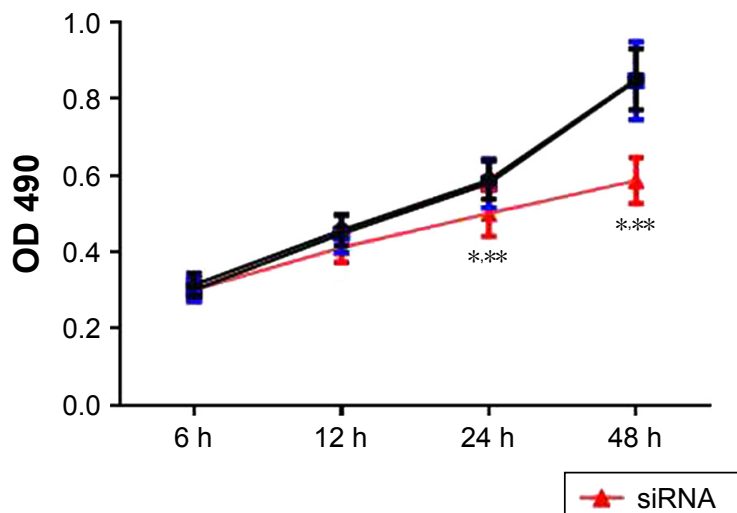

HL-60
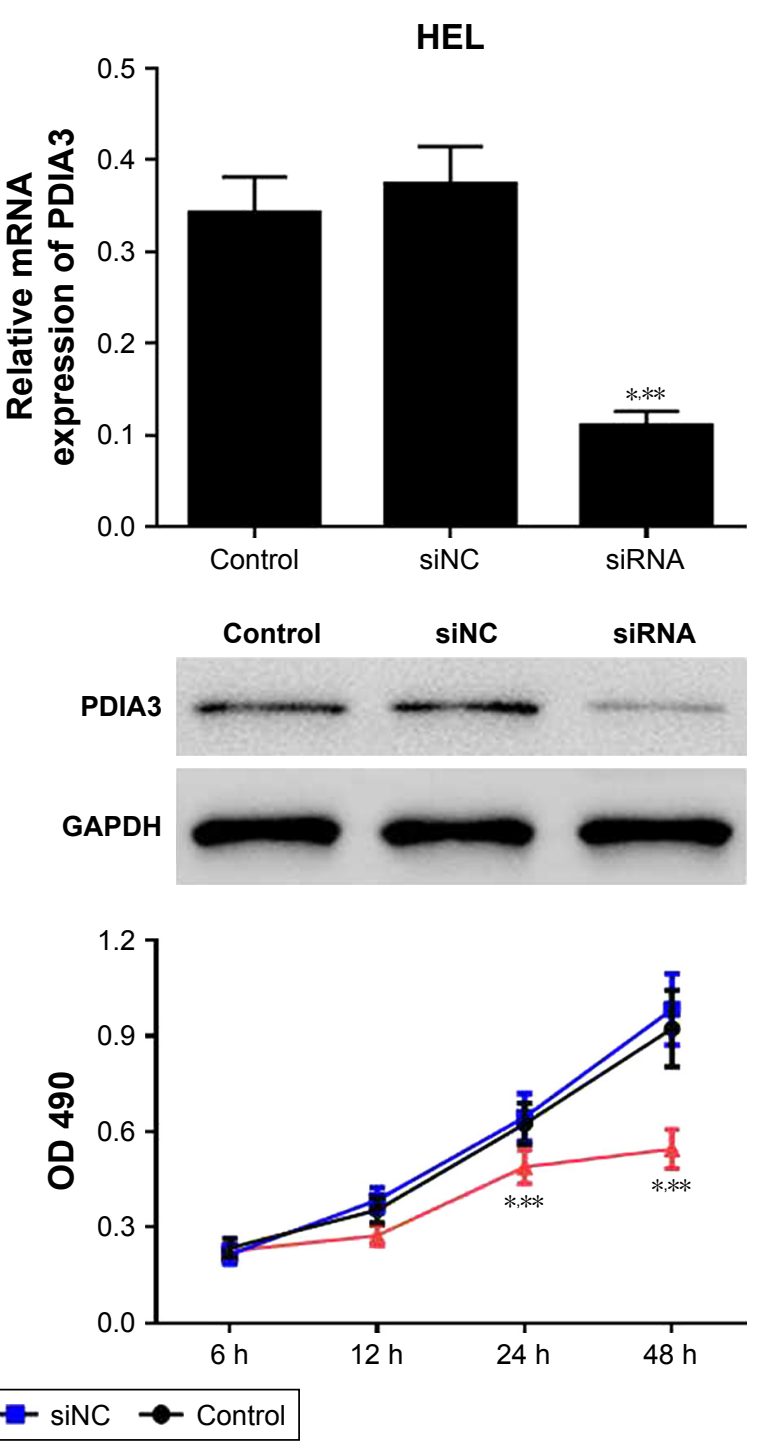

Figure 2 PDIA3 siRNA inhibits cell proliferation.

Notes: HL-60 and HEL cells were transfected with PDIA3 siRNA, and mRNA expression and protein expression of PDIA3 were examined by RT-PCR (A) and Western blot analysis (B), respectively. (C) Cell proliferation of control, siNC, and siRNA groups of HL-60 and HEL cells was identified by MTT assay. $* P<0.0 \mathrm{I}$ compared with the control cells; **P $<0.01$ compared with the siNC cells. Data are expressed as the mean $\pm S D, n=6$.

Abbreviation: GADPH, glyceraldehyde 3-phosphate dehydrogenase. 


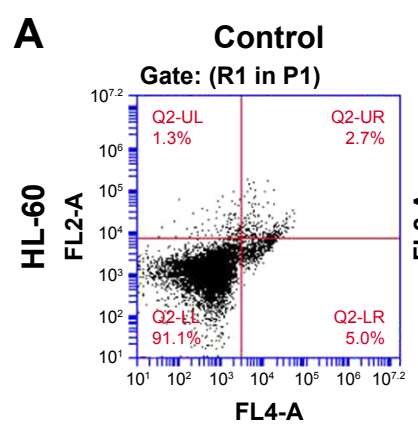

B

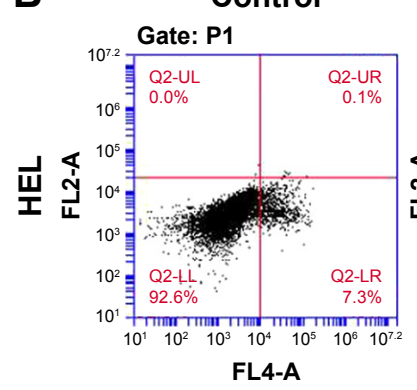

C
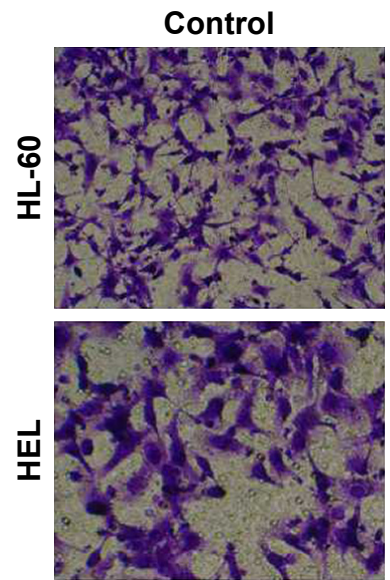

D
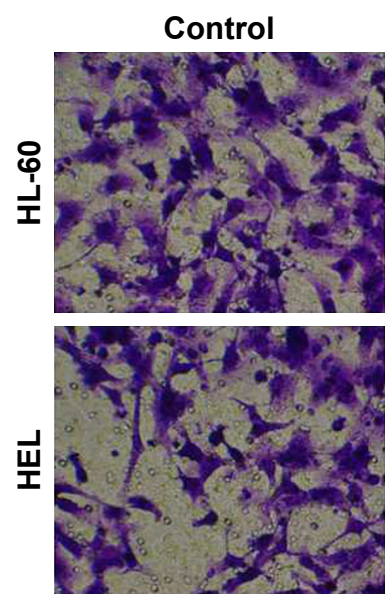

siNC

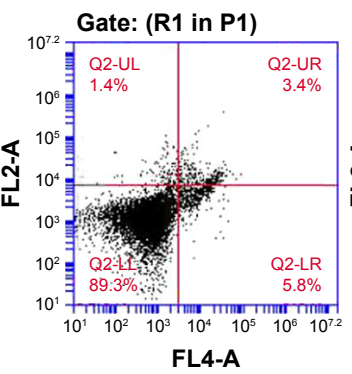

FL4-A

siNC

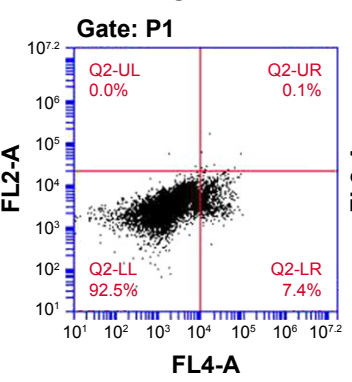

SiNC
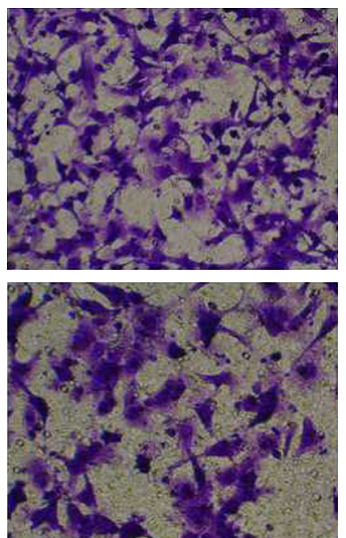

siNC
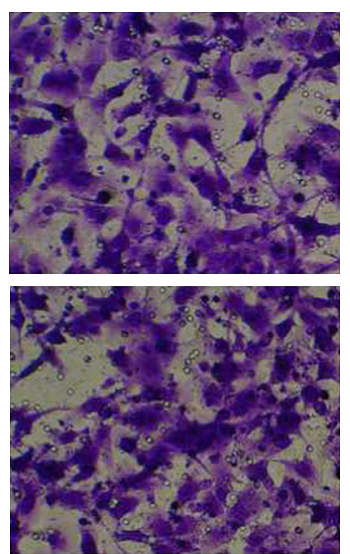

SiRNA
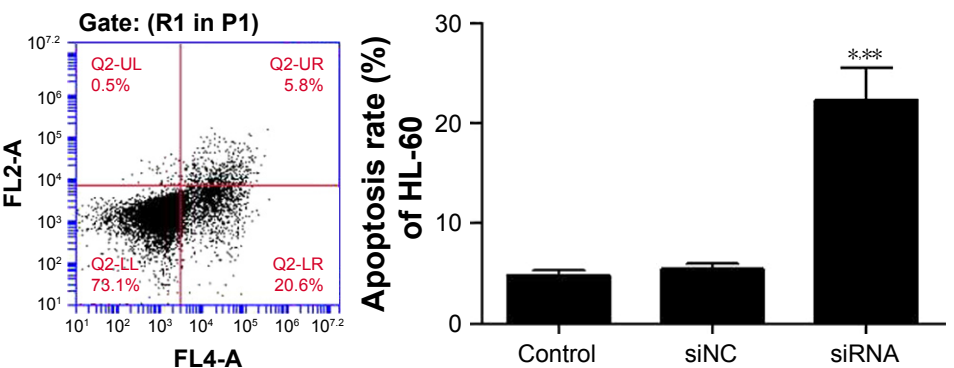

SIRNA
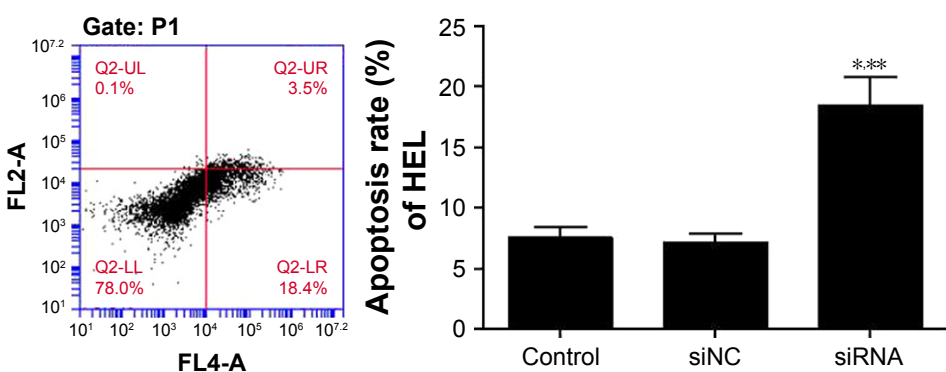

SIRNA
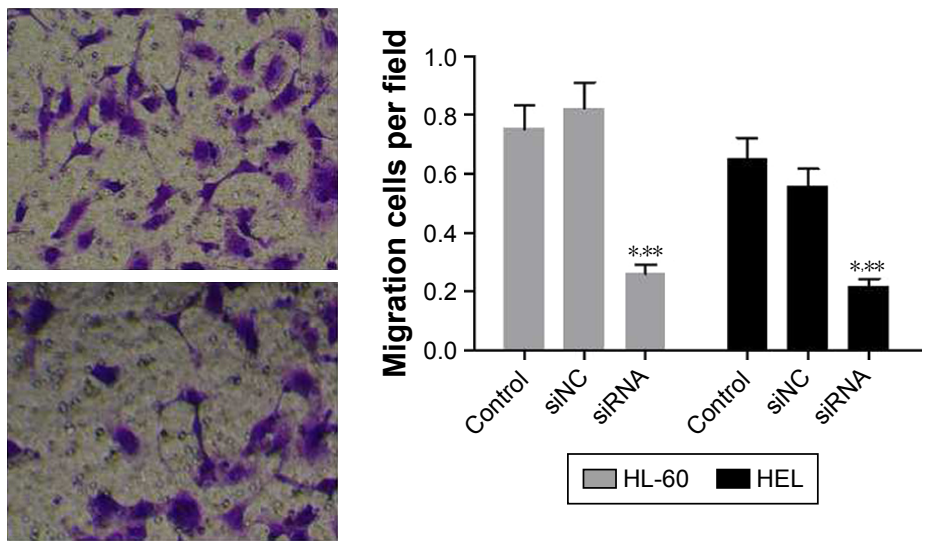

$\square \mathrm{HL}-60 \quad \square \mathrm{HEL}$

Figure 3 PDIA3 siRNA induces cell apoptosis and inhibits cell migration and invasion.

Notes: (A and B) After PDIA3 siRNA transfection for $48 \mathrm{~h}$, cell apoptosis of HL-60 and HEL cells was identified by flow cytometry. (C and D) Transwell assay was used to identify the migration and invasion of HL-60 and HEL cells following PDIA3 siRNA treatment for $48 \mathrm{~h}$. $* P<0.01$ compared with the control cells; $* * P<0.0 \mathrm{I}$ compared with the siNC cells. Data are expressed as the mean $\pm S D, n=6$. Magnification $\times 400$. 
The transwell invasion test results are presented in Figure 3C. Transfection of PDIA3 siRNA resulted in a significantly weakened invasive ability of HL-60 and HEL cells as compared with that of the control and siNC groups $(P<0.01)$. Consistently, as shown in Figure 3D, the migration ability of HL-60 and HEL cells in the PDIA3 siRNA group was dramatically declined than that of control and mock cells $(P<0.01)$. Our data suggested that interference of PDIA3 reduces the migration and invasion ability of HL-60 and HEL cells.

\section{PDIA3 siRNA regulated the expression of MATI, PCNA, caspase-9, RhoC, and CDKI}

To elucidate the potential mechanism underlying PDIA3induced cell invasion and migration, the expression levels of PCNA, MAT1, caspase-9, RhoC, and CDK1, which are closely associated with cell proliferation, invasion, and migration, were measured by Western blotting. Figure 4A and $B$ reveals a significant decrease in protein levels of PCNA, CDK1, RhoC, and MAT1, but an evident increase in caspase-9 expression in the PDIA3 siRNA group compared with those in the control and siNC groups $(P<0.01)$. The results indicated that PDIA3 siRNA may exert an antitumor effect via regulation of MAT1, PCNA, caspase-9, RhoC, and CDK1 expression.

\section{PDIA3-associated pathways in AML}

To further explore the role of PDIA3 in AML, we performed GSEA in AML samples with higher PDIA3 expression versus those with lower PDIA3 expression based on TCGA dataset. Our data implied that higher PDIA3 expression in AML samples was positively correlated with KEGG oxidative phosphorylation and amino sugar and nucleotide sugar metabolism pathways (Figure 5A).

To further validate the GSEA results, we then detected the protein expression of oxidative phosphorylation (COX2 and COX5A) and amino sugar and nucleotide sugar metabolism pathway-related proteins (UGP2 and UAP1) in PDIA3-silenced AML cells. The levels of detected protein were significantly decreased in both HEL and HL-60 cells (Figure 5B) after the downregulation of PDIA3.

\section{Effect of PDIA3 siRNA on MAPK signaling}

Accumulating evidence suggests that MAPK signaling plays a crucial role in carcinoma growth and metastasis.
We then evaluated the phosphorylation (p-) of P38, JNK, and ERK by Western blotting. As shown in Figure 6A and B, PDIA3 siRNA significantly decreased the p-P38, p-JNK, and p-ERK expression compared with that in the control and siNC groups $(P<0.01)$. The results implied that PDIA3 siRNA inhibited cell proliferation, invasion, and migration, and induced cell apoptosis, possibly by suppressing MAPK phosphorylation.

\section{Discussion}

AML is the most common type of acute leukemia in adults, with over 20,000 new diagnoses each year in the US. ${ }^{8,9}$ AML usually results from a cooperative effect of several independent mutations that lead to cell malignant transformation. Several oncogenes that are most often activated in AML have been characterized. ${ }^{10-12}$ Interference of the involved critical genes or proteins is considered as a favorable strategy. Located at 15 qn5, PDIA3 is reported to be a PDI. Increased PDIA3 expression was associated with an elevated Ki-67 index, indicating increased cancer cell proliferation, reduction in apoptotic cell death, and poor prognosis. Recent studies have shown that PDIA3 regulates cell invasiveness in cervical cancer and that a high level of PDIA3 is associated with a low patient survival rate. ${ }^{13}$ As reported in our study, notable elevated expression of PDIA3 in bone marrow tissues of myeloid leukemia was found, proposing a new question about the role of PDIA3 in human AML development and progression. Consequently, the subsequent work was designed to explore the effect of PDIA3 siRNA on tumor migration and invasion in human AML.

The present study focused on the biological role of PDIA3 in AML. Notably, upregulated expression of PDIA3 was found in tissues of AML patients when compared with those of the healthy controls. HL-60 and HEL cell lines were further investigated for high expression of PDIA3. Our research revealed that PDIA3 siRNA significantly suppressed proliferation, invasion, and migration of AML HL-60 and HEL cells. PDIA3 siRNA also induced apoptosis of AML cells. Moreover, we demonstrated that the above effects may be mediated through the inhibition of PCNA, CDK1, RhoC, and MAT1 expression, and the promotion of caspase-9 expression at the protein level. The phosphorylation of P38, JNK, and ERK was also inhibited by PDIA3 siRNA in AML cells. Taken together, these findings indicated that PCNA, CDK1, MAT1, RhoC, and caspase-9, and MAPK signaling play a pivotal role in the process of antiproliferation, antiinvasion, antimigration, and apoptosis-induction triggered by PDIA3 siRNA. 
A

HL-60

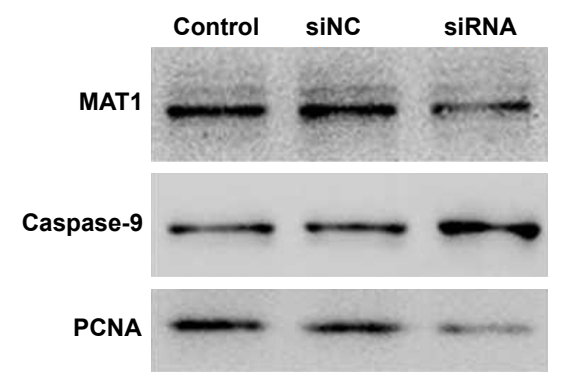

\section{HL-60}
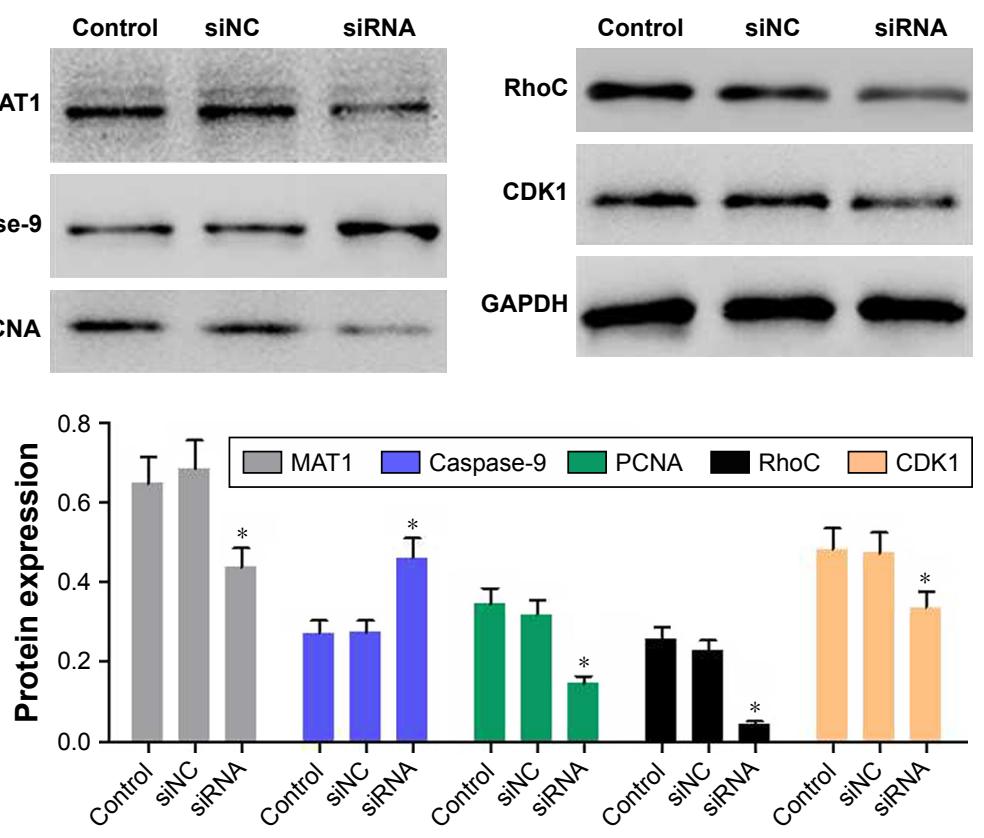

\section{B}

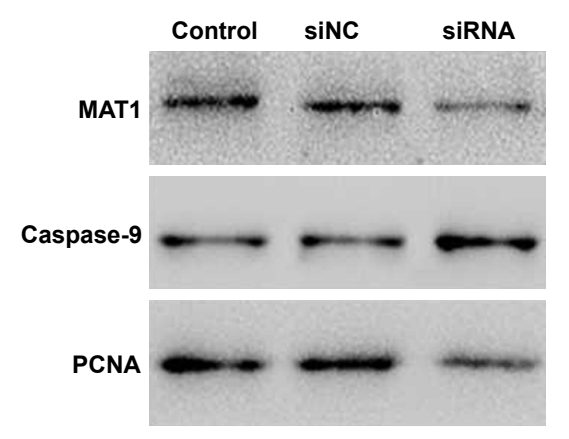

HEL
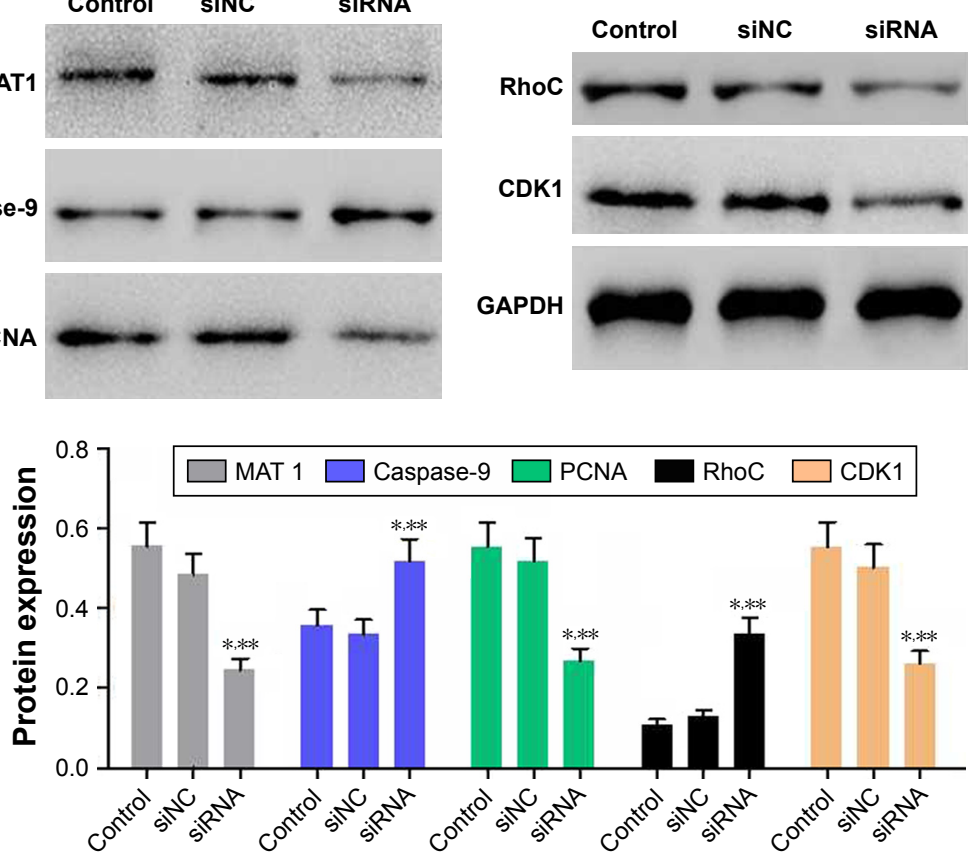

Figure 4 Effect of PDIA3 siRNA on expressions of PCNA, CDKI, MATI, RhoC, and caspase-9.

Notes: After PDIA3 siRNA treatment for $48 \mathrm{~h}$, Western blot was utilized to analyze the protein levels of PCNA, CDKI, MATI, RhoC, and caspase-9 in HL-60 (A) and HEL (B) cells. GAPDH was also detected as the control of sample loading. *P $<0.01$ compared with the control cells; **P $<0.01$ compared with the siNC cells. Data are expressed as the mean $\pm S D, n=6$.

Abbreviation: GADPH, glyceraldehyde 3-phosphate dehydrogenase.

We first found that siRNA of PDIA3 effectively inhibited cell proliferation and induced cell apoptosis. PCNA is a cyclin that is generally expressed in the $\mathrm{S}$ phase of the cell cycle. ${ }^{14}$ CDK1 is one of the core regulatory proteins in cell cycle regulation. ${ }^{14}$ In our results, siRNA of PDIA3 significantly suppressed the expression of PCNA and CDK1. Caspase-9 is a crucial element in cell apoptosis ${ }^{15}$ and is upregulated, notably, by PDIA3 siRNA treatment. Tumor cell invasion and migration is the essential process in the metastasis of malignant tumor. MTA1 is a stress response protein that is 
A

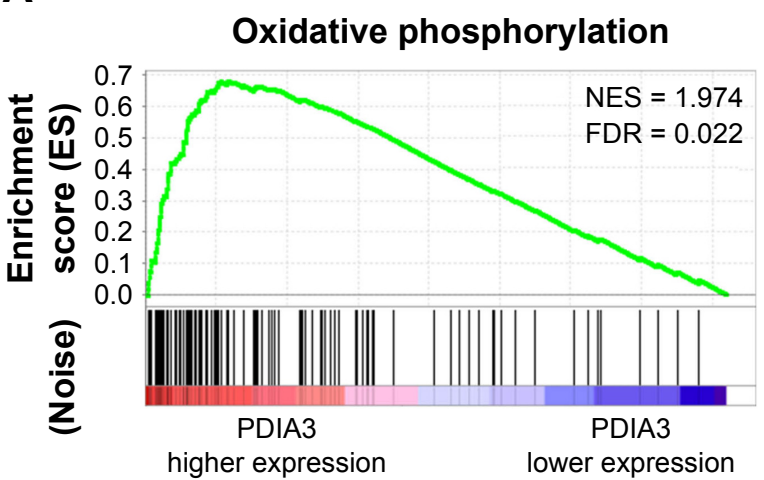

Amino sugar and nucleotide sugar metabolism

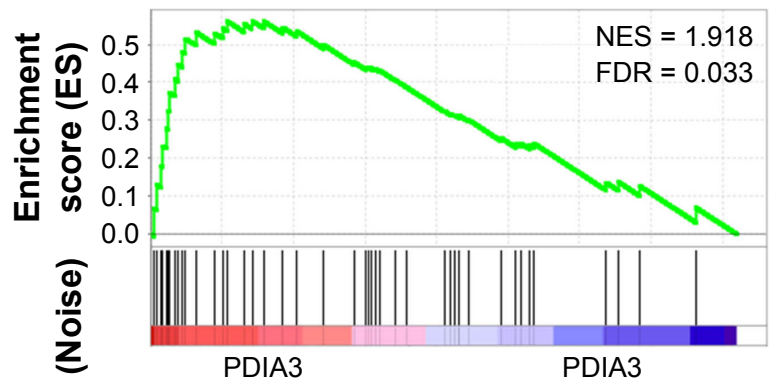

higher expression

lower expression
B
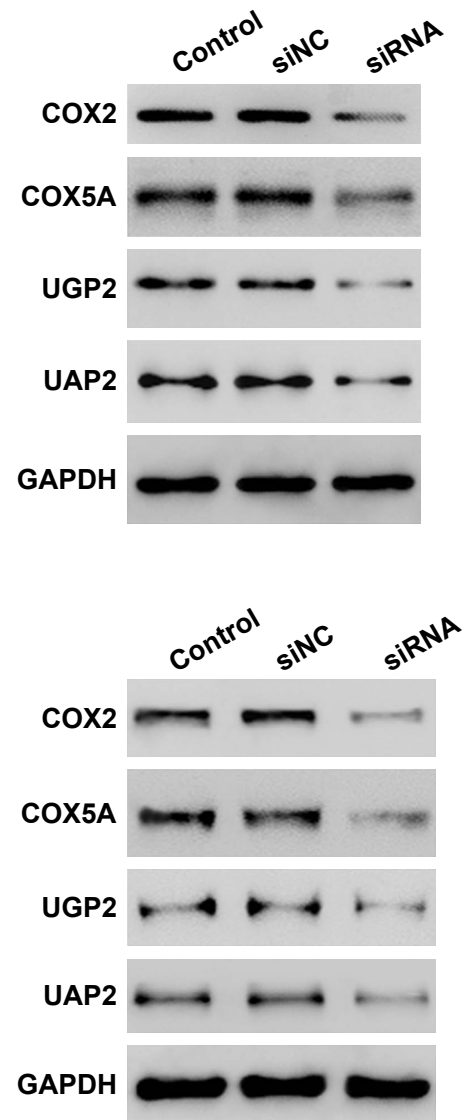

HL-60

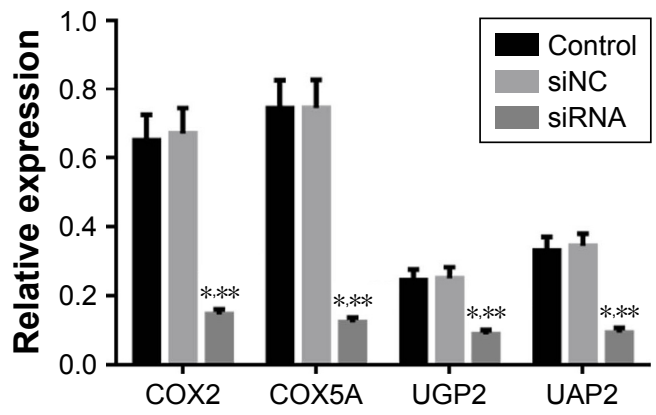

HEL

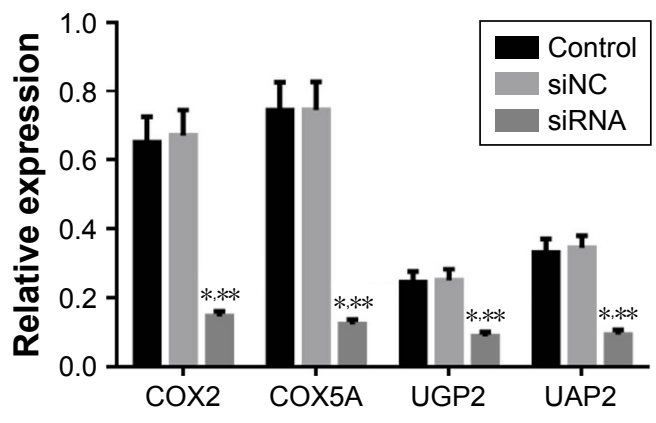

Figure 5 Mechanisms of how PDIA3 exerts its function in AML.

Notes: (A) Gene set enrichment analysis in AML patients with higher PDIA3 expression versus lower PDIA3 expression based on the Cancer Genome Atlas datasets. Oxidative phosphorylation and amino sugar and nucleotide sugar metabolism pathways have the strongest association with higher expression of PDIA3. (B) Western blot was utilized to analyze the protein levels of COX2, COX5A, UGP2, and UAP2 in HL-60 and HEL cells. GAPDH was also detected as the control of sample loading. $* P<0.01$ compared with the control cells; ${ }^{* * P}<0.01$ compared with the siNC cells. Data are expressed as the mean $\pm S D, n=6$.

Abbreviations: GADPH, glyceraldehyde 3-phosphate dehydrogenase; NES, normalized enrichment score.

upregulated in various stress-related situations, and cancer cells mostly live in a stressful environment. To cope with all these stresses, the expression of MTA1, which plays the role of a master regulator of gene expression, is upregulated and helps cancer cells to survive and migrate out of their original site. ${ }^{16}$ It is reported that RhoC overexpression can give rise to cell invasion through regulation of cytoskeletal proteins and destruction of polarity in cancer cells. Our study showed that PDIA3 siRNA significantly inhibited the expression of MTA1 and RhoC.

To further explore the role of PDIA3 in AML, we performed GSEA of AML samples. In the present study, GSEA data indicated that higher PDIA3 expression was positively correlated with KEGG oxidative phosphorylation and amino 
A

HL-60
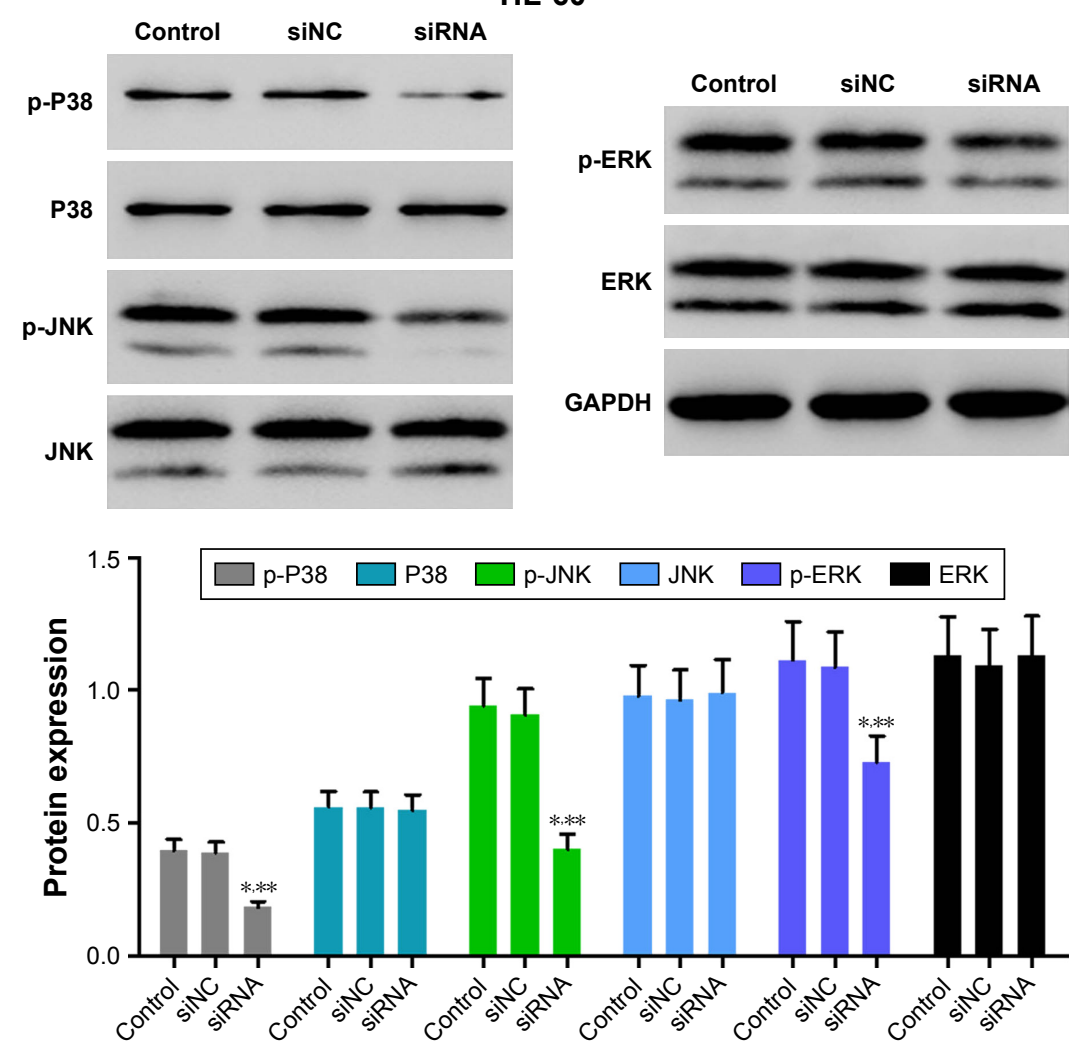

B

HEL
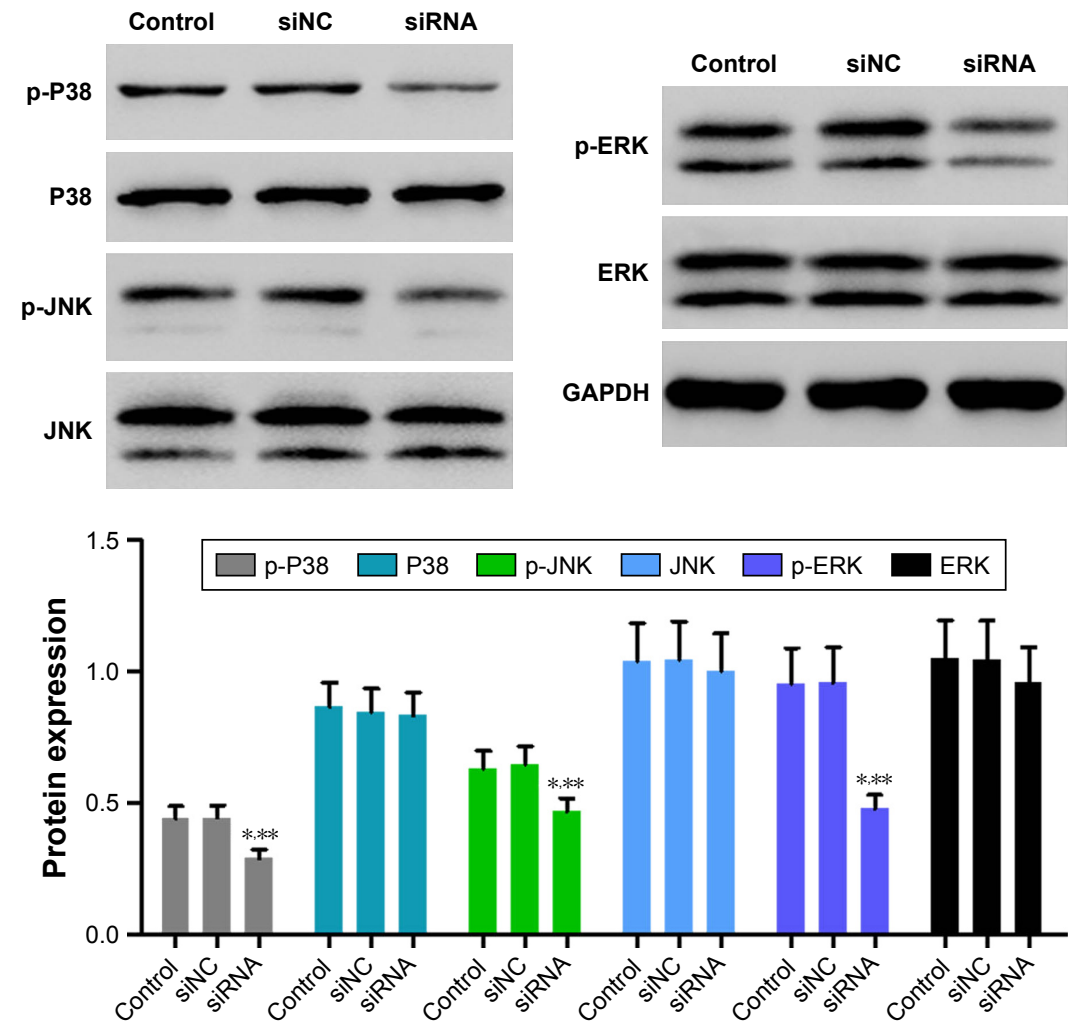

Figure 6 Effect of PDIA3 siRNA on MAPK signaling.

Notes: After PDIA3 siRNA treatment for 48 h, Western blot was utilized to analyze the protein levels of p-P38/P38, p-JNK/JNK, and p-ERK/ERK in HL-60 (A) and HEL (B) cells. GAPDH was also detected as the control of sample loading. $* P<0.01$ compared with the control cells; $* * P<0.01$ compared with the siNC cells. Data are expressed as the mean $\pm S D, n=6$.

Abbreviation: GADPH, glyceraldehyde 3-phosphate dehydrogenase. 
sugar and nucleotide sugar metabolism pathways in AML samples. AML occurrence and progression depend upon oxidative phosphorylation and amino sugar and nucleotide sugar metabolism pathways. ${ }^{17,18}$

The expressions of oxidative phosphorylation-related factors $\left(\mathrm{COX} 2\right.$ and $\left.\mathrm{COX} 5 \mathrm{~A}^{19}\right)$ and amino sugar and nucleotide sugar metabolism pathway-related factors (UGP2 and UAP1 ${ }^{20}$ ) were significantly suppressed by PDIA3 knockdown. These data suggested the roles of PDIA3 on oxidative phosphorylation and amino sugar and nucleotide sugar metabolism, accounting for AML carcinogenesis. MAPK pathway is one of the most extensively studied protein kinase pathways, which can be subdivided into three subtypes, including ERK1/2, P38, and JNK. ${ }^{21,22}$ As an important signaling pathway in mammals, MAPK signaling pathway is stimulated by cytokines and involved in cell proliferation, differentiation, apoptosis, adhesion, invasion, metastasis, immunoregulation, and other important biological processes in AML. ${ }^{23,24}$

Collectively, we have demonstrated that PDIA3 siRNA effectively enhanced apoptosis and suppressed proliferation, invasion, and migration of AML HL-60 and HEL cells by regulating proliferation and metastasis-related proteins, oxidative phosphorylation and amino sugar, and nucleotide sugar metabolism pathways, and MAPK signaling. We will confirm these findings at in vivo level. These findings may elucidate the molecular mechanism underlying the effect of PDIA3 siRNA on cell growth, apoptosis, migration, and invasion in AML, and lead to the development of therapeutic approaches for the disease.

\section{Acknowledgment}

This work was supported by Scientific Research Program of Shanghai Municipal Commission of Health and Family Planning (program number 201540178).

\section{Disclosure}

The authors report no conflicts of interest in this work.

\section{References}

1. Izzi V, Lakkala J, Devarajan R, et al. Expression of a specific extracellular matrix signature is a favorable prognostic factor in acute myeloid leukemia. Leuk Res Rep. 2017;9:9-13.

2. Ganapule A, Nemani S, Korula A, et al. Allogeneic stem cell transplant for acute myeloid leukemia: evolution of an effective strategy in India. J Glob Oncol. 2017;3(6):773-781.

3. Fontana MC, Marconi G, Feenstra JDM, et al. Chromothripsis in acute myeloid leukemia: biological features and impact on survival. Leukemia. Epub 2017 Dec 18.
4. Chen J, Doroudi M, Cheung J, Grozier AL, Schwartz Z, Boyan BD. Plasma membrane Pdia3 and VDR interact to elicit rapid responses to $1 \alpha, 25(\mathrm{OH})(2) \mathrm{D}(3)$. Cell Signal. 2013;25(12):2362-2373.

5. Takata H, Kudo M, Yamamoto T, et al. Increased expression of PDIA3 and its association with cancer cell proliferation and poor prognosis in hepatocellular carcinoma. Oncol Lett. 2016;12(6):4896-4904.

6. Chay D, Cho H, Lim BJ, et al. ER-60 (PDIA3) is highly expressed in a newly established serous ovarian cancer cell line, YDOV-139. Int J Oncol. 2010;37(2):399-412.

7. Ramos FS, Serino LT, Carvalho CM, et al. PDIA3 and PDIA6 gene expression as an aggressiveness marker in primary ductal breast cancer. Genet Mol Res. 2015;14(2):6960-6967.

8. Sun Y, Huskey RL, Tang L, et al. Adverse effects of intravenous vancomycin-based prophylaxis during therapy for pediatric acute myeloid leukemia. Antimicrob Agents Chemother. 2018;62(3):e01838-e01917.

9. Sexauer AN, Tasian SK. Targeting FLT3 signaling in childhood acute myeloid leukemia. Front Pediatr. 2017;5:248.

10. Stein EM, Walter RB, Erba HP, et al. A phase 1 trial of vadastuximab talirine as monotherapy in patients with CD33-positive acute myeloid leukemia. Blood. 2018;131(4):387-396.

11. Zhou JD, Ma JC, Zhang TJ, et al. High bone marrow ID2 expression predicts poor chemotherapy response and prognosis in acute myeloid leukemia. Oncotarget. 2017;8(54):91979-91989.

12. Guo Q, Luan J, Li N, et al. MicroRNA-181 as a prognostic biomarker for survival in acute myeloid leukemia: a meta-analysis. Oncotarget. 2017;8(51):89130-89141.

13. Chung H, Cho H, Perry C, Song J, Ylaya K, Lee H, Kim JH. Downregulation of ERp57 expression is associated with poor prognosis in early-stage cervical cancer. Biomarkers. 2013;18(7):573-579.

14. Jia Z, Zhao W, Fan L, Sheng W. The expression of PCNA, c-erbB-2, p53, ER and PR as well as atypical hyperplasia in tissues nearby the breast cancer. J Mol Histol. 2012;43(1):115-120.

15. Kim B, Srivastava SK, Kim SH. Caspase- 9 as a therapeutic target for treating cancer. Expert Opin Ther Targets. 2015;19(1):113-127.

16. Sandhu C, Connor M, Kislinger T, Slingerland J, Emili A. Global protein shotgun expression profiling of proliferating mcf-7 breast cancer cells. J Proteome Res. 2005;4(3):674-689.

17. Whang-Peng J, Henderson ES, Knutsen T, Freireich EJ, Gart JJ. Cytogenetic studies in acute myelocytic leukemia with special emphasis on the occurrence of Ph1 chromosome. Blood. 1970;36(4):448-457.

18. Zapf B, Carpenter RJ 3rd, Snyder GG. Ulcerating tumor of tonsil and neck mass as occurrence of acute myelocytic leukemia. Otolaryngol Head Neck Surg. 1981;89(4):560-563.

19. Corazao-Rozas P, Guerreschi P, André F, et al. Mitochondrial oxidative phosphorylation controls cancer cell's life and death decisions upon exposure to MAPK inhibitors. Oncotarget. 2016;7(26):39473-39485.

20. Moolten FL. Tumor chemosensitivity conferred by inserted herpes thymidine kinase genes: paradigm for a prospective cancer control strategy. Cancer Res. 1986;46(10):5276-5281.

21. Eriksson M, Peña-Martínez P, Ramakrishnan R, et al. Agonistic targeting of TLR1/TLR2 induces p38 MAPK-dependent apoptosis and NFKB-dependent differentiation of AML cells. Blood Adv. 2017; 1(23):2046-2057.

22. Mak PY, Mak DH, Mu H, et al. Apoptosis repressor with caspase recruitment domain is regulated by MAPK/PI3K and confers drug resistance and survival advantage to AML. Apoptosis. 2014;19(4):698-707.

23. Choi CH, Xu H, Bark H, Lee TB, Yun J, Kang SI, Oh YK. Balance of NF-kappaB and p38 MAPK is a determinant of radiosensitivity of the AML-2 and its doxorubicin-resistant cell lines. Leuk Res. 2007; 31(9):1267-1276.

24. Li Q, Huai L, Zhang C, et al. Icaritin induces AML cell apoptosis via the MAPK/ERK and PI3K/AKT signal pathways. Int J Hematol. 2013; 97(5):617-623. 
OncoTargets and Therapy

\section{Publish your work in this journal}

OncoTargets and Therapy is an international, peer-reviewed, open access journal focusing on the pathological basis of all cancers, potential targets for therapy and treatment protocols employed to improve the management of cancer patients. The journal also focuses on the impact of management programs and new therapeutic agents and protocols on The manuscript management system is completely online and includes a very quick and fair peer-review system, which is all easy to use. Visit http://www.dovepress.com/testimonials.php to read real quotes from published authors.

Submit your manuscript here: http://www.dovepress.com/oncotargets-and-therapy-journal 